\title{
EDITORIAL AND COMMENT Ethics in Conflict: Moral Distress as a Root Cause of Burnout
}

\author{
Elizabeth Dzeng, MD, PhD, MPH and Robert M. Wachter, MD
}

Division of Hospital Medicine, Department of Medicine, University of California, San Francisco, San Francisco, CA, USA.

J Gen Intern Med 35(2):409-11

DOI: $10.1007 / \mathrm{s} 11606-019-05505-6$

(c) Society of General Internal Medicine 2019

I $\mathrm{n}$ this commentary, we argue that moral distress and professional ethical dissonance are root causes of burnout; the way forward requires attention to the social and ethical dimensions of professional practice. The Physician's Charter on Medical Professionalism, a charter for the new millennium written in 2002 through a partnership between the American Board of Internal Medicine, the American College of Physicians, and the European Federation of Internal Medicine, identified several threats to physician professionalism including technology, market forces, healthcare system strain, and broader sociological shifts in the role of physicians in society. ${ }^{1}$ The Charter described how these factors challenged physicians' ability to adhere to the values of professionalism.

Adding to these challenges, the medical profession is now experiencing a crisis of burnout. Burnout, characterized by emotional exhaustion, depersonalization (i.e., cynicism), and reduced personal accomplishment, has been linked to poorer quality of care and decreased patient safety. ${ }^{2}$ Doctors who experience burnout (and currently approximately 50\% do) have a two-fold increase in suicidal ideation. ${ }^{3}$ Tragically, around 400 doctors commit suicide every year.

Prevailing explanations on the causes of burnout have focused on long work hours, the rapid adoption of electronic health records (EHRs), and grinding administrative tasks. We believe that the wellsprings of discontent run deeper. We see evidence of an insidious moral distress resulting from physicians' inability to act in accord with their individual and professional ethical values due to institutional and societal constraints. These constraints have been exacerbated by changes in healthcare and society, changes that go beyond those identified in the Charter. This discontent amplifies a growing rift between the profession's ethical ideals and reality.

Moral distress' most pernicious expression is in moral apathy - a moral cynicism derived from feelings of powerlessness, which provides a rich medium for the growth of burnout. To address physician burnout, we must look beyond mechanistic explanations, examining why many physicians feel unable to exercise ethical agency that is so central to their professional identity as providers of ethical

Published online October 30, 2019 care. While others have described this phenomenon as "moral injury," 4 we believe that rooting this discussion within the context of moral distress and ethics provides a valuable framework and tool to understand and develop potential solutions against burnout.

Everyday insults to professional values map onto the three pillars of the Physician Charter. They illustrate how healthcare systems create ethical tensions, both large and small, that lead to moral distress and burnout. Below are some examples.

\section{PRINCIPLE OF PRIMACY OF PATIENT WELFARE}

The consumerization of medicine has challenged the physician's imperative to act in a patient's best interest (beneficence). Physicians are buffeted by conflicting pressures to reduce costs in some settings while in others, to raise institutional or individual incomes by prescribing or referral practices. Something as simple as RVU-driven throughput incentives may impede meaningful conversations with patients or disincentivize exploration of health concerns that extend beyond the patient's reason for visiting.

\section{PRINCIPLE OF PATIENT AUTONOMY}

Physicians strive to offer treatment plans that respect patients' values and preferences. However, everyday systemic factors challenge physicians' ability to reconcile respecting autonomy with maximizing patient welfare. One example is when physicians feel compelled to provide potentially harmful or futile treatments, because there is no advance directive or family members disagree, subjecting a patient to perceived futile care near the end-of-life results in moral distress. Conversely, a patient's refusal of life-sustaining treatment or desire to seek assistance in dying in states where it is legal may also create moral distress. Both situations conflict with the precept to "do no harm" and create tension with the value of respect for persons. Physicians may deploy coping mechanisms of dehumanization and detachment to deal with their perceived powerlessness as moral agents. They then absolve themselves of responsibility for the negative consequences of care by reasoning that systemic constraints offered them no leeway, further contributing to moral apathy. 


\section{PRINCIPLE OF SOCIAL JUSTICE}

The emphasis on healthcare as a business rather than a human right has created a cultural milieu that works against patient welfare. Examples abound. From the skyrocketing costs of critically important medications to the unaffordable costs of insurance and high out-of-pocket payments, physicians work within a system that regularly violates the Physician's Charter. Physicians aware of the social determinants of health regularly battle the effects of a gutted social safety net, resulting in egregious disparities in the quality of care. They feel powerless to address systemic threats to their patients' health, such as inadequate medication drug coverage resulting in a preventable stroke, inadequate screening resulting in a delayed cancer diagnosis, and a lack of housing and food resulting in markedly worse outcomes in people who are homeless.

\section{THE WAY FORWARD REQUIRES SITUATING THE DISCUSSION AND POTENTIAL SOLUTION IN ETHICS}

The clinician-ethicist Richard Gunderman wrote in the Atlantic (2014), "professional burnout is the sum total of hundreds and thousands of tiny betrayals of purpose, each one so minute that it hardly attracts notice." These betrayals of purpose with their attendant pressure to engage in practices that contradict ethical ideals contribute powerfully to today's crisis of burnout and disillusionment.

Moral distress can have a lasting impact on the culture of medicine as a whole. Individual moments of moral distress lead to alienation, detachment, and loss of empathy, which in turn produce a culture of cynical care devoid of meaning. Indeed, the phenomenon of individual moral distress can be mapped onto a larger and systemic ethical malaise within the medical profession. A deeper understanding of the underlying sources and mechanisms of discontent provides new avenues for interventions to improve the well-being of healthcare professionals and the culture of medicine as a whole.

As with many complex social phenomena, many of the forces that have led to growing rates of physician burnout are well-intended. The digitization of medicine and residency duty hour reductions, for example, are appropriate responses to real problems. However, interventions to prevent or mitigate burnout must also acknowledge the deeper underlying pathologies of values in conflict and moral distress. They require the courage to question the broader market-based trends and systemic changes that so often pit physicians' professional ideals against today's reality.

Solutions for burnout should focus on enabling clinicians to act according to their professional values. Healthcare organizations will need to prioritize ethics at the highest level of hospital leadership and embed ethical values into the core of their organization's mission. Creating an organizational ethics aligned with medicine's professional values requires responsive ethics and other leadership committees that empower physicians to exercise their moral agency to act ethically, rather than what would be safest for risk management or best for a short-term bottom line. We should also remain cognizant of the unintended consequences of interventions against burnout that create values conflicts such as restrictions on resident work hours leading to perceptions of unprofessionalism and compromised patient safety among trainees. Most importantly, we should strive towards reforming our inherently unethical system that privileges market-driven mandates to prioritize shareholder profit over providing care in the patient's best interest.

Encouraging physicians to advocate for issues that directly impact their patients, such as the recent \#stayinyourlane efforts regarding gun control or advocating for universal healthcare coverage, empowers physicians to stand up for patient welfare and social justice and adds their voices to the many others working towards social and political change. This change however is a long game; strategies to combat moral distress and burnout must be established in the meantime. Perhaps most fundamentally, ethical awareness and ethics education must be strengthened for both medical learners as well as seasoned professionals, to provide the foundational ethical knowledge, skills, and moral courage to practice medicine in ways consistent with their professional values. While ethics education and a focus on ethics cannot solve these very real systemic strains, it can help arm physicians with the language and framework of ethics to more successfully navigate these challenges on an individual and institutional level. For example, in addition to focusing on reducing residency duty hours to alleviate burnout, residency leadership could also focus on interventions that improve trainees' abilities to provide ethical care such as increasing resources and support around the discharge of homeless patients or providing resources and training around end-of-life care rooted in an ethical lens. One of us (E.D.) found that institutions whose cultures and policies prioritized autonomy over beneficence encouraged trainee behaviors that reflected a simplistic understanding of autonomy to mean offering limitless choice while eschewing the important role of physician recommendation in decision-making $^{5}$. We hypothesize that institutional prioritization of beneficence could encourage a more nuanced understanding of autonomy and help transcend ethical dilemmas where autonomy and beneficence appear to conflict.

Burnout is a complex and multidimensional problem. The degree to which it is a symptom of a larger ethical malaise has been underemphasized. Creating more efficient support systems and teaching resilience skills will go only so far. Taking the Professionalism Charter seriously as a guide to sources of moral distress can help re-ground the medical profession in an authentic moral framework.

Acknowledgments: The author would like to thank Christine Cassel, MD; Amy Markowitz, JD; and Alexander K. Smith, MD (all from the University of California, San Francisco) for helpful feedback on drafts of this editorial.

Corresponding Author: Elizabeth Dzeng, MD, PhD, MPH; Division of Hospital Medicine, Department of Medicine, University of California, San Francisco, San Francisco, CA, USA (e-mail: liz.dzeng@ucsf.edu). 
Funding Information Dr. Dzeng is supported by the Center for Advancing Translational Sciences of the NIH under Award Number KL2TROO1870 as well as NIH grant 1R03AG060098.

\section{Compliance with Ethical Standards:}

Conflict of Interest: Dr. Wachter reports that he is a member of the Lucian Leape Institute of the Institute for Healthcare Improvement (no compensation except travel expenses); has a contract to UCSF from the Agency for Healthcare Research and Quality to edit a patient-safety website; receives royalties from Lippincott Williams \& Wilkins and McGraw-Hill for writing/editing several books; receives stock options for serving on the board of Accuity Medical Management Systems; receives a yearly stipend for serving on the board of The Doctors Company and on the Global Advisory Board for Teledoc; serves on the scientific advisory boards for Amino.com, PatientSafe Solutions, and EarlySense (for which he receives stock options); consults with Commure (for which he receives a stipend and stock options) and Forward (stock options); has a small royalty stake in CareWeb, a hospital communication tool developed at UCSF; has given $>200$ talks (a few to for-profit entities including Nuance, GE, Health Catalyst, AvaCare) for which he has received honoraria; and holds the Benioff Endowed Chair in Hospital Medicine from Marc and Lynne Benioff and the Holly Smith Distinguished Professorship in Science and Medicine at UCSF. Dr. Dzeng reports no conflicts of interest.

Disclaimer: The content is solely the responsibility of the authors and does not necessarily represent the official views of the NIH.

\section{REFERENCES}

1. ABIM Foundation, Foundation A, Medicine EF of I. Medical Professionalism in the New Millenium: A Physician Charter. Ann Intern Med 2002;136(3):243-246. doi:https://doi.org/10.7326/0003-4819-136-3200202050-00012

2. Panagioti $\mathbf{M}$, Geraghty $\mathbf{K}$, Johnson $\mathbf{J}$, et al. Association between Physician Burnout and Patient Safety, Professionalism, and Patient Satisfaction: A Systematic Review and Meta-analysis. JAMA Intern Med 2018;178(10):1317-1330. doi:https://doi.org/10.1001/jamainternmed. 2018.3713

3. Shanafelt TD, West CP, Sinsky C, et al. Changes in Burnout and Satisfaction With Work-Life Integration in Physicians and the General US Working Population Between 2011 and 2017. Mayo Clin Proc 2019;1-14. doi:https://doi.org/10.1016/j.mayocp.2018.10.023

4. Talbot S, Dean W. Physicians aren't 'burning out.' They're suffering from moral injury. STAT. https://www.statnews.com/2018/07/26/physiciansnot-burning-out-they-are-suffering-moral-injury/. Accessed July 4, 2019.

5. Dzeng E, Colaianni A, Roland M, et al. Influence of Institutional Culture and Policies on Do-Not-Resuscitate Decision Making at the End of Life. JAMA Intern Med 2015;175(5):812-819. doi:https://doi.org/10.1001/ jamainternmed.2015.0295

Publisher's Note Springer Nature remains neutral with regard to jurisdictional claims in published maps and institutional affiliations. 